\title{
Comparison of a combined quantum mechanics/interatomic potential function approach with its periodic quantum-mechanical limit: Proton siting and ammonia adsorption in zeolite chabazite
}

\author{
Martin Brändle ${ }^{\text {a) }}$ and Joachim Sauer ${ }^{\text {b) }}$ \\ Humboldt-Universität zu Berlin, Institut für Chemie, Arbeitsgruppe Quantenchemie, Jägerstr. 10/11, \\ D-10117 Berlin, Germany \\ Roberto Dovesi \\ Department of Inorganic, Physical and Materials Chemistry, University of Torino, Via Pietro Giuria 5, \\ I-10125 Torino, Italy \\ Nicholas M. Harrison \\ CLRC Daresbury Laboratory, Daresbury, WA4 4AD Warrington, United Kingdom
}

(Received 8 June 1998; accepted 14 September 1998)

\begin{abstract}
Comparison is made between a combined quantum mechanics/interatomic potential function approach (QM-Pot) and its fully quantum-mechanical limit, $a b$ initio calculation applying periodic boundary conditions. The Hartree-Fock (HF) method is combined with ab initio-parametrized ion pair shell model potential functions. The CRYSTAL code is employed for the periodic Hartree-Fock calculations. The same double-/valence triple-zeta polarization basis sets are used in both the approaches. The proton siting and ammonia adsorption in a high-silica acidic zeolite catalyst, $H$-chabazite $\left(\mathrm{Si} / \mathrm{Al}=11\right.$, space group $P 1$, unit cell $\left.\mathrm{H}-\mathrm{AlO}_{2}\left[\mathrm{SiO}_{2}\right]_{11}\right)$ are examined. The combined QM-Pot relative stabilities and reaction energies deviate from the periodic full QM results by 4-9 $\mathrm{kJ} / \mathrm{mol}$ only, which demonstrates the power of our combined approach. This conclusion is also supported by comparison of the electrostatic potential inside the zeolite pore, calculated from the periodic wave function and by the QM-Pot approach. Framework oxygen O1 is found to be the preferred proton site and on interaction with $\mathrm{NH}_{3}$ the proton is predicted to move to $\mathrm{NH}_{3}$ yielding $\mathrm{NH}_{4}^{+}$. The $\mathrm{NH}_{4}^{+}$surface species is coordinated to two framework oxygen atoms. It is by 30-35 $\mathrm{kJ} / \mathrm{mol}$ more stable than the neutral adsorption complex of $\mathrm{NH}_{3}$. Evidence is produced that the failure of previous periodic $\mathrm{HF}$ calculations to predict a stable $\mathrm{NH}_{4}^{+}$ion is due to the limitations of the minimum basis set used. (C) 1998 American Institute of Physics. [S0021-9606(98)70847-5]
\end{abstract}

\section{INTRODUCTION}

In spite of tremendous progress in computer technology and code development modeling the structure and predicting the reactivity of large chemical systems is still a challenge for $a b$ initio quantum chemistry. One strategy is to limit the quantum-mechanical description to the part of special interest and to describe its environment by interatomic potential functions. There is a very large number of slightly different implementations of such methods for different types of systems (see Ref. 1 for a recent review). Typical systems are solute molecules in a solvent (or complexes of the solute with a few first shell solvent molecules), where the solutesolvent and solvent-solvent interactions are described by simple intermolecular potential functions. ${ }^{1-3}$ Other examples are active sites of enzymes in their protein environment ${ }^{4}$ or the core of transition metal catalysts ${ }^{5}$ in their environment of large ligands. We are particularly interested in the active sites of solid catalysis. ${ }^{6-9}$ Specifically, we are interested in

\footnotetext{
a) Present address: ETH Zürich, Laboratorium für anorganische Chemie, Universitätstr. 6, CH-8092 Zürich, Switzerland.

${ }^{b)}$ Author to whom correspondence should be addressed. Electronic mail: js@qc.ag-berlin.mpg.de
}

schemes that allow structure optimizations and are not merely including selected contributions of the total interaction with the environment for a given structure, such as embedding in a set of point charges. Mostly, the approximations that are unavoidable in these hybrid schemes are judged on experimental data, but internal consistency checks are better suited. Rarely it has been possible (see Refs. 5 and 10, for examples) to make explicit comparison with the full quantum-mechanical solution for the large system within the same set of quantum-mechanical approximations, i.e., using the same method and applying the same basis set.

Because of the periodicity of our large systems we are able to make such comparison by applying periodic boundary conditions. We study the proton form of zeolite chabazite, an acidic catalyst which-on adsorption of ammoniadonates a proton yielding the ammonium form of the zeolite. For this reaction we present both combined quantum mechanics/interatomic potential function (QM-Pot) and periodic Hartree-Fock ${ }^{11,12}$ (HF) calculations using a double-/ valence triple-zeta polarization basis set. Comparison between the QM-Pot approach and its periodic HF limit is also made for the relative stabilities of chabazites protonated at different oxygen sites of the zeolite framework. The periodic HF calculations are too costly for structure optimizations. 


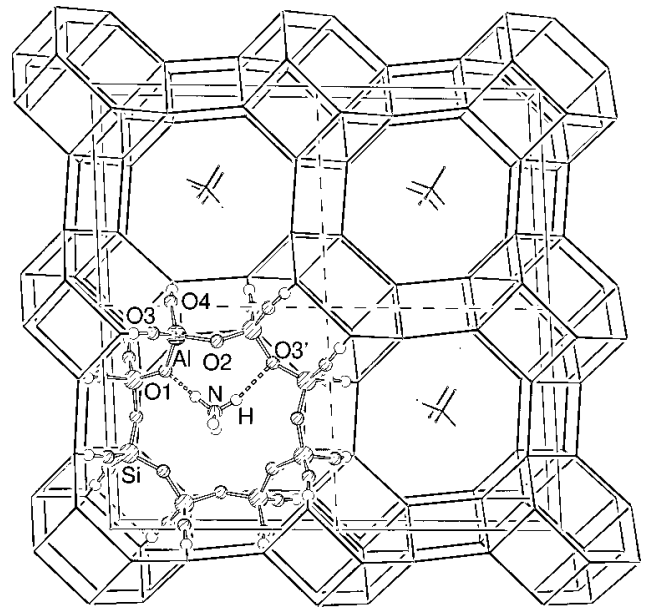

FIG. 1. Eight-ring model embedded in $2 \times 2 \times 1$ supercell of $\mathrm{NH}_{4}$-chabazite. Numbering of $\mathrm{O}$ atoms according to Ref. 40. Similar models were embedded in the $\mathrm{NH}_{3}$ loaded, protonated, and deprotonated chabazite frameworks.

Therefore, we optimize all structures by the QM-Pot method and make the comparison between periodic HF and combined QM-Pot calculations at the level of single point energies. We include also a comparison of the electrostatic potentials inside the zeolite cavity calculated by both methods.

\section{SYSTEM STUDIED AND PREVIOUS CALCULATIONS}

The chabazite framework has been selected as an example of a zeolite catalyst for the following reasons. From the computational point of view it is a favorable case since it contains only $12 \mathrm{TO}_{2}$ units per unit cell $(\mathrm{T}=\mathrm{Si}, \mathrm{Al})$. On the other hand, chabazites are interesting catalysts because of their recently demonstrated ability to selectively convert methanol into $\mathrm{C}_{2}-\mathrm{C}_{4}$ olefines. ${ }^{13}$ Their acidity has been characterized by $\mathrm{NH}_{3}$ microcalorimetry. ${ }^{14}$ Figure 1 shows the chabazite structure. It shows pores formed by eightmembered silicate rings and cavities large enough to accommodate adsorbates like ammonia, methanol, or $\mathrm{C}_{2}-\mathrm{C}_{4}$ hydrocarbons. Chabazites can be prepared with varying $\mathrm{Al}$ content. To facilitate comparison with other zeolites, ${ }^{8,9}$ we use a high silica chabazite of framework composition $\mathrm{Si}_{11} \mathrm{AlO}_{24}$ /uc. Every $\mathrm{Al}$ atom creates a negative framework charge which is compensated by a proton, and an acidic Brønsted site, $\mathrm{Si}-\mathrm{O}(\mathrm{H})-\mathrm{Al}$, is created. Due to the high symmetry of the framework the site at which one $\mathrm{Al}$ per unit cell is introduced is unique, but the proton can be attached to any of the four oxygen positions linked to the Al site. The present study deals with the relative stability of different proton sites and with ammonia adsorption on a single acid site in the eight-ring pore.

The heat of ammonia adsorption measured by microcalorimetry or temperature-programmed desorption (TPD) characterises the acid strength of solids. On adsorption of $\mathrm{NH}_{3}$ on acidic zeolites, $\mathrm{Z}-\mathrm{OH}$, the acidic proton is transferred onto ammonia and ammonium ions are formed which interact with the negatively charged surface site of the zeolite, $\mathrm{ZO}^{-} \cdot \mathrm{NH}_{4}^{+} \cdot{ }^{15-18}$ Hence, the energy of adsorption of $\mathrm{NH}_{3}$ depends not only on the energy of deprotonation of the zeolite (DP), but also on the proton affinity of $\mathrm{NH}_{3}(\mathrm{PA})$ and the binding energy of the ammonium ion onto the negatively charged surface (ion pair binding energy IP), as shown in the thermodynamic cycle below.

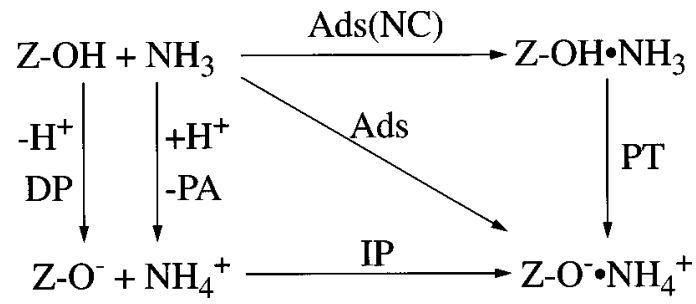

For gas-phase molecules, acidity is clearly defined as heat of deprotonation. Such data are available from mass or pulsed ion cyclotron resonance spectroscopy which, however, cannot be applied to surface hydroxyl groups. Quantum chemical calculations instead provide a direct access to the heat of deprotonation of surface hydroxyl groups. ${ }^{8,9,19,20}$

Due to its small unit cell, the chabazite structure has already been subject of several theoretical studies applying both periodic boundary conditions ${ }^{21-24}$ and different embedded cluster and free space cluster calculations. ${ }^{19,25-27}$ For example, periodic DFT methods using plane-wave basis sets were employed to examine the initial step of the methanolto-gasoline conversion. ${ }^{22,23}$ Among the studies of $\mathrm{NH}_{3}$ adsorption and the proton transfer reaction in this zeolite is a periodic HF calculation ${ }^{21}$ and a calculation using Pisani's Green's function embedding scheme. ${ }^{27}$ Due to the computational limitations, the calculations were done on a structure with $\mathrm{R} 3$ space group symmetry $(\mathrm{Si} / \mathrm{Al}=3)$ and applied a STO-3G basis set which is not appropriate for studying proton transfer reactions as explained in Ref. 19.

\section{COMBINED QUANTUM MECHANICS-INTERATOMIC POTENTIAL FUNCTION CALCULATIONS}

\section{A. Summary of the QM-Pot approach}

The embedding scheme ${ }^{6}$ we use decomposes the energy of the entire system $(S)$ into contributions of an inner part $(I)$ containing the active site in question, of an outer part $(O)$ and of an interaction term $(I-O)$. Only the inner part is treated quantum mechanically. The energy of the outer part and all interactions between the inner and the outer part are evaluated at the level of interatomic potentials. If the inner part is chemically bonded to the outer region, the partitioning leads to dangling bonds which need to be saturated by terminating $\mathrm{H}$ atoms, also called link atoms $(L)$. The $L$ atoms and the inner part form the cluster, $C=I+L$. The energy of the total system can be approximated by the subtraction scheme

$$
E_{\mathrm{QM}-\mathrm{Pot}}(S)=E_{\mathrm{QM}}(C)+E_{\mathrm{Pot}}(S)-E_{\mathrm{Pot}}(C),
$$

which involves only energies that can be obtained from straightforward application of available codes to welldefined systems. The assumption is made that

$$
\Delta=-E_{\mathrm{QM}}(L)-E_{\mathrm{QM}}(L-I)+E_{\mathrm{Pot}}(L)+E_{\mathrm{Pot}}(L-I) \approx 0,
$$


which will be valid if the interatomic potential function mimicks the quantum-mechanical potential-energy surface for the link atoms $L$ and their interaction with the inner part $L-I$. Hence, the subtraction scheme [Eq. (1)] eliminates approximately the contribution from the terminating atoms which are not part of the real solid. The price we have to pay is that the interatomic potential has to be defined also for the cluster itself including the link atoms. Interatomic potential parameters for link atoms are in general not available from empirical sources. Therefore, the use of potential functions fitted to $a b$ initio data becomes necessary. Hence, differently from other embedded cluster schemes, in our scheme there is no direct influence of the charge distribution of the outer part on the wave function of the cluster. The latter is different from the wave function of the gas-phase cluster only by way of structure changes the cluster experiences when it is embedded in the outer part. However, energy contributions due to mutual polarization between the inner and the outer regions are included at the level of the interatomic shell model potential. Charge-transfer effects are included only to the extent they are mimicked by the link atoms.

The forces on the nuclei used for structure optimization are obtained as

$$
\begin{aligned}
& F_{\alpha, \mathrm{QM}-\mathrm{Pot}}(S)=F_{\alpha, \mathrm{QM}}(C)+F_{\alpha, \mathrm{Pot}}(S)-F_{\alpha, \mathrm{Pot}}(C), \\
& \alpha \in I, \\
& F_{\beta, \mathrm{QM}-\mathrm{Pot}}(S)=F_{\beta, \mathrm{Pot}}(S), \quad \beta \in O .
\end{aligned}
$$

The terminating atoms are not moved independently, but their positions are given by the positions of the atoms of the corresponding bond in the crystal. This creates additional contributions to the forces on these atoms. ${ }^{6}$

The reaction energy for a general reaction $R \rightarrow P$ can approximately be expressed as 7,8

$$
\Delta E \approx \Delta E_{\mathrm{QM} / / \mathrm{QM}-\mathrm{Pot}}+\Delta E_{\mathrm{lr} / / \mathrm{QM}-\mathrm{Pot}},
$$

with

$$
\Delta E_{\mathrm{QM} / / \mathrm{QM}-\mathrm{Pot}}=E_{\mathrm{QM}}\left(C_{P}\right)-E_{\mathrm{QM}}\left(C_{R}\right)
$$

and

$$
\begin{aligned}
\Delta E_{\mathrm{lr} / / \mathrm{QM}-\mathrm{Pot}}= & E_{\mathrm{Pot}}\left(S_{P}\right)-E_{\mathrm{Pot}}\left(S_{R}\right)-\left(E_{\mathrm{Pot}}\left(C_{P}\right)\right. \\
& \left.-E_{\mathrm{Pot}}\left(C_{R}\right)\right) .
\end{aligned}
$$

Subscripts $R$ and $P$ refer to reactants and products, respectively. The notation " $/ / \mathrm{QM}$-Pot" means "at the structure obtained by the combined QM-Pot method." In deriving Eq. (5) the assumptions are made that (i) the cluster is chosen such that the reaction changes the inner part of the system only (ii) the structure of the outer part is about the same for $P$ and $R$, and (iii) the cluster is large enough that all shortrange terms of the interatomic potentials vanish between the active site and the cluster boundary. This way of writing Eq. (5) stresses that the combined QM-Pot approach has two effects:

(i) The quantum-mechanical contribution to the reaction energy is calculated at structures of the reactant and product cluster models that have been obtained by the combined
QM-Pot approach. Compared to free space clusters nuclear relaxation of the embedded clusters is limited due to the presence of the embedding force field.

(ii) The interatomic potential functions describing the interaction between the inner and the outer region provide a long-range (lr) correction to the calculated reaction energy.

A recent implementation ${ }^{6,28}$ couples the quantum chemical code TURBOMOLE ${ }^{29}$ with the General Utility Lattice Program (GULP) ${ }^{30}$ using interatomic potentials for periodic calculations. In the present application we use the Hartree-Fock method for the QM part and an $a b$ initio(HF)-parametrized shell model potential for zeolites ${ }^{31}$ and their interaction with $\mathrm{NH}_{3}$ and $\mathrm{NH}_{4}{ }^{+}$.

\section{B. Technical details}

The Hartree-Fock calculations use double-zeta basis sets for silicon, aluminum, and hydrogen, and valence triplezeta basis sets for the oxygen and nitrogen atoms. For the $\mathrm{Si}$, $\mathrm{Al} / \mathrm{O}, \mathrm{N} / \mathrm{H}$ atoms the $(11 s, 7 p / 9 s, 5 p / 4 s)$ Gaussian basis sets of Huzinaga ${ }^{32}$ were contracted according to the pattern $\{521111,4111\} /\{51111,311\} /\{31\}$. Polarization functions were added to all atoms with exponents $0.4(\mathrm{Si}), 0.3(\mathrm{Al}), 1.2(\mathrm{O})$, $1.0(\mathrm{~N})$, and $0.8(\mathrm{H})$. This is the basis set used in previous studies on zeolites from this laboratory. ${ }^{6-9,19}$ It is denoted $\mathrm{T}(\mathrm{O}, \mathrm{N}) \mathrm{DZP}$.

The cutout was made such that the clusters terminate with $\mathrm{OH}$ groups. For the terminating $\mathrm{OH}$ groups fixed distances $r_{\mathrm{OH}}$ of 94.5 and $94.0 \mathrm{pm}$ were used if bonded to $\mathrm{Si}$ and $\mathrm{Al}$, respectively. These values were obtained by free cluster optimizations with the same basis set. ${ }^{33}$ All SCF calculations were carried out in $C_{1}$ symmetry.

Energy and gradient calculations for the interatomic potential description of the entire periodic system $(S)$ and the embedded cluster $(C)$ [see Eqs. (1), (3), and (4)] were performed using the GULP code. ${ }^{30}$ The interatomic potential functions fitted to $a b$ initio Hartree-Fock data of gas-phase cluster models of zeolite catalysts ${ }^{7,31}$ adopt the dipolar shell model introduced by Dick and Overhauser. ${ }^{34}$ It splits the ion into a core and a shell connected by a harmonic spring, and thus, accounts empirically for the polarization of the ions in an electric field. The electrostatic energy is evaluated by standard Ewald summation techniques for all cores and shells and the shell positions are optimized to yield the lowest energy. For the summation of short-range interactions a cutoff radius of $10 \AA$ is chosen.

It is convenient to optimize first the structure of the entire periodic system in constant pressure mode applying the shell model potential only. This has the advantage that the positions of the atoms in the outer region are already substantially relaxed and optimization cycles can be saved in the following QM-Pot part. In addition, it allows a broad screening for preferred adsorbate positions at low computational expense. The implementation of the QM-Pot scheme used ${ }^{28}$ performs constant volume optimizations and does not exploit space group symmetry. Thresholds of $0.01 \mathrm{~kJ} / \mathrm{mol}$ (energy) and $1 \mathrm{~kJ} /(\mathrm{mol} \AA)$ (largest component of energy gradient) were taken as convergence criterion. In the case of deprotonated zeolites, the excess charge occuring in each unit cell 
TABLE I. Lattice constants of chabazites protonated at sites O1-O4, of ammonia loaded chabazites, ${ }_{a}^{\mathrm{a}, \mathrm{b}}$ and of the deprotonated chabazite obtained with shell model potential calculations. Si/Al=11. Cell sizes in Angstrøm, angles in degree.

\begin{tabular}{cccccccr}
\hline \hline Lattice constants & $\mathrm{O} 1-\mathrm{H}$ & $\mathrm{O} 2-\mathrm{H}$ & $\mathrm{O} 3-\mathrm{H}$ & $\mathrm{O} 4-\mathrm{H}$ & $\mathrm{Z}-\mathrm{O}(1) \mathrm{H} \cdots \mathrm{NH}_{3}{ }^{\mathrm{a}}$ & $\mathrm{Z}-\mathrm{O}^{-} \cdot \mathrm{NH}_{4}^{+}$ & Anion \\
\hline$a$ & 9.449 & 9.418 & 9.414 & 9.475 & 9.439 & 9.428 & 9.490 \\
$b$ & 9.342 & 9.371 & 9.374 & 9.380 & 9.350 & 9.312 & 9.341 \\
$c$ & 9.395 & 9.380 & 9.368 & 9.342 & 9.385 & 9.366 & 9.373 \\
$\alpha$ & 93.82 & 94.28 & 94.37 & 94.30 & 92.88 & 93.56 & 94.26 \\
$\beta$ & 95.44 & 96.72 & 93.94 & 94.90 & 95.04 & 94.72 & 95.38 \\
$\gamma$ & 94.62 & 94.40 & 94.07 & 94.32 & 93.24 & 92.80 & 94.06 \\
\hline \hline
\end{tabular}

${ }^{a}$ Neutral adsorption complex, $\mathrm{Z}-\mathrm{O}(1) \mathrm{H} \cdots \mathrm{NH}_{3}$, cf. Fig. 2, bottom.

${ }^{\mathrm{b}}$ Ion pair adsorption complex, $\mathrm{Z}-\mathrm{O}^{-} \cdot \mathrm{NH}_{4}^{+}$, cf. Fig. 2, top.

for the periodic system was neutralized by immersing the framework into a homogeneous background charge distribution.

Deprotonation energies are calculated with respect to removal of a single proton in an otherwise periodic array of bridging hydroxyl groups in the periodic framework. Similarly, the binding energy of the ion pair is calculated with respect to the insertion of a single free $\mathrm{NH}_{4}^{+}$ion into an anionic $\mathrm{NH}_{4}^{+}$-loaded chabazite, in which one site is free of $\mathrm{NH}_{4}^{+}$. This is achieved ${ }^{8}$ by use of a macroscopic approximation for the interaction of charged defects in a lattice, ${ }^{35}$ which compensates for the interaction of the protons or adsorbates within the zeolite. Regular arrays of protons and $\mathrm{NH}_{4}^{+}$ions immersed in a homogeneous compensating background charge were constructed with the same geometry they have in the optimized chabazites. The distance between them is about $940 \mathrm{pm}$ (vide infra, Table I). For $\mathrm{NH}_{4}^{+}$, the interaction energy was determined with respect to a single distorted adsorbate having the same structure as in the zeolite. The screening of the interaction by the surrounding dielectric crystal medium is considered by dividing the interaction energy by the static dielectric constant of $\mathrm{H}-\mathrm{CHA}$ or $\mathrm{NH}_{4}-\mathrm{CHA}$ obtained from a shell model calculation. From such calculations for $\alpha$-quartz, ${ }^{31}$ we know that the calculated dielectric constants are smaller by eight percent than the measured values. The screened interaction energies for the protons and the ammonium ions are large, -81 and -83 $\mathrm{kJ} / \mathrm{mol}$, respectively. They are included in the QM-Pot deprotonation and ion-pair binding energies. The difference between them introduces a deviation of $2 \mathrm{~kJ} / \mathrm{mol}$ in the sum of the reaction energies over the thermodynamic cycle (Scheme I). It is probably caused by the approximation of the dielectric constants. For $\mathrm{NH}_{3}-\mathrm{CHA}$, the screened interaction between the $\mathrm{NH}_{3}$ molecules is neglibible, $-0.5 \mathrm{~kJ} / \mathrm{mol}(\mathrm{QM}-$ Pot) and $-0.2 \mathrm{~kJ} / \mathrm{mol}$ (periodic HF). Hence, it is neglected when calculating the physisorption energy, $\Delta E_{\text {ads }}(\mathrm{NC})$.

\section{PERIODIC HARTREE-FOCK CALCULATIONS}

The CRYSTAL program ${ }^{11,12}$ was used for the periodic Hartree-Fock calculations. To allow comparison with the combined QM-Pot results the same basis set, T(O,N)DZP, was employed as in the QM part of the QM-Pot calculations. This basis set, designed for molecules, possesses diffuse valence functions. The lowest exponents are 0.096 a.u. ${ }^{-2}$ for $\mathrm{Si}$ and 0.076 a.u. $^{-2}$ for Al. In periodic HF calculations such diffuse functions may lead to serious SCF energy convergence problems due to two main sources of error: ${ }^{36}$ (i) $\mathrm{Nu}$ merical errors made in the evaluation of the individual mono- and bielectronic integrals. These errors accumulate in the SCF energy, which involves a sevenfold summation over three lattice vectors and the four AO indices in the bielectronic integrals of the Coulomb and exchange series. For large basis sets with diffuse functions the number of integrals and the concomitant error accumulation increase dramatically. (ii) Most seriously, inappropriate lattice sum truncations. In the CRYSTAL code, proper convergence of the Coulomb series is achieved by the combination of nuclearelectron and electron-electron interactions and by use of Ewald summation techniques. ${ }^{11,12,37}$ Proper convergence of the exchange series is most difficult to achieve since the exchange contribution has no counterpart of opposite sign as the Coulomb term has and, therefore, must converge by itself. The techniques and the corresponding overlap and penetration thresholds used to control the accuracy of the real space summations have been described elsewhere. ${ }^{11,12}$ For the diffuse functions present, the tolerances described by the ITOL $n$ parameters ${ }^{11,12}$ for the Coulomb and exchange series

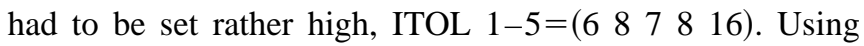
these criteria, an accuracy of the total SCF energy of $10^{-5}-10^{-4}$ a.u./atom can be expected. Correspondingly, the criteria for convergence of the SCF energy and the SCF eigenvalues were set to $10^{-3}$ and $10^{-5}$ a.u., respectively. To achieve better convergence, Fock matrix mixing of $30 \%$ and a nonlocked level shifting ${ }^{38}$ of 0.1 a.u. was allowed. In all calculations reported below, convergence of the SCF energy was better than $1.9 \cdot 10^{-4}$ a.u. Hence, the calculated reaction energies are accurate to about $1 \mathrm{~kJ} / \mathrm{mol}$. No use of symmetry could be made since the space group of all structures was $P 1$. For Brillouin zone integration eight $k$-points located on the corners of the triclinic Brillouin zone were used. The eigenvalues of the overlap matrices $\mathbf{S}(\mathbf{k})$ were larger than $10^{-3}$, indicating that linear dependencies among basis functions are not a problem. Calculations were performed on an 8-CPU R8000/90 MHz SGI PowerChallenge employing the direct-SCF, replicated-data version of CRYSTAL95 ${ }^{12}$ linked with the TCGMSG library ${ }^{39}$ for message passing. For the largest system, a primitive unit cell of $\mathrm{NH}_{4}^{+}$-chabazite comprising 41 atoms, 771 basis functions, and 369 atomic shells, the 
TABLE II. Relative stabilities and absolute and relative deprotonation energies $(\mathrm{kJ} / \mathrm{mol})$ for chabazites $(\mathrm{Si} / \mathrm{Al}=11)$ protonated at the four different sites $\mathrm{O} 1-\mathrm{O} 4$

\begin{tabular}{lrrrr}
\hline \hline \multicolumn{1}{c}{ Relative stabilities } & O1-H & O2-H & O3-H & O4-H \\
\hline Shell model only, Pot & 1.7 & 18.8 & 15.7 & 0.0 \\
Embedded 2T model, QM-Pot & 0.0 & 22.2 & 16.4 & 8.7 \\
Periodic HF//QM-Pot $^{\mathrm{a}}$ & 0.0 & 17.0 & 12.9 & 12.6 \\
& & & & \\
Deprotonation energy & & & 1258 & 1266 \\
Embedded 2T model, QM-Pot & 1277 & 1255 & -19 & -11 \\
Relative values & 0 & -22 & & \\
\hline \hline
\end{tabular}

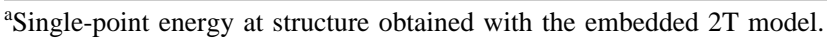

calculation time for one SCF cycle was about 449 min., all CPUs being active. Convergence of the SCF energy was achieved after eight cycles.

\section{RESULTS AND DISCUSSION}

\section{A. Proton sites in chabazites}

Figure 1 shows the chabazite structure and the numbering ${ }^{40}$ of oxygen atoms. Note that other studies, e.g., Ref. 41, use a different numbering of $\mathrm{O}$ atoms. To determine the most stable of the four possible nonequivalent proton positions, constant pressure shell model optimizations were carried out. Table I gives lattice constants of the optimized structures. A recent powder neutron diffraction study of $\mathrm{H}-$ SSZ-13 (high-silica chabazite, $\mathrm{Si} / \mathrm{Al}=16$, space group R-3m) reports lattice constants $a=9.281 \AA$ and $\alpha=94.27^{\circ}{ }^{41}$ Our lattice constants for $\mathrm{H}-\mathrm{CHA}$ are slightly larger, $a$ $=9.34-9.48 \AA$.

Table II contains the relative stabilities of the frameworks protonated at sites $\mathrm{O} 1-\mathrm{O} 4$. The optimizations with the shell model potential alone yield the proton at position $\mathrm{O} 4$ pointing into the six-membered ring as the most stable, closely followed by position $\mathrm{O} 1-\mathrm{H}$ which is less stable by $1.7 \mathrm{~kJ} / \mathrm{mol}$. Combined QM-Pot calculations on di-tetrahedra models $(2 \mathrm{~T})$ embedded at the four bridging hydroxyl groups provide an improved description of the acid site (Table II shows the energies and Table III selected structure data). The relative stability of positions $\mathrm{O} 1-\mathrm{H}$ and $\mathrm{O} 4-\mathrm{H}$ is reversed, and the proton is predicted to bind preferentially at position O1.

The deprotonation energies obtained from the embedded $2 \mathrm{~T}$ models follow the same order as the relative stabilities (Table II, bottom), but their relative values deviate by up to $3 \mathrm{~kJ} / \mathrm{mol}$ from the above relative stabilities (in absolute

TABLE III. Selected bond lengths (pm) and angles (deg) of di-tetrahedra models with $\mathrm{O}(1)$ to $\mathrm{O}(4)$ as bridging atoms embedded in H-chabazite ( $\mathrm{Si} /$ $\mathrm{Al}=11)$.

\begin{tabular}{lrrrr}
\hline \hline & $\mathrm{O} 1$ & $\mathrm{O} 2$ & $\mathrm{O} 3$ & \multicolumn{1}{c}{$\mathrm{O} 4$} \\
\hline $\mathrm{O}-\mathrm{Hb}$ & 95.7 & 95.7 & 95.8 & 96.0 \\
$\mathrm{Si}-\mathrm{O}$ & 170.6 & 169.7 & 169.6 & 171.6 \\
$\mathrm{Al}-\mathrm{O}$ & 190.7 & 186.9 & 187.0 & 192.1 \\
$\mathrm{Al}-\mathrm{Hb}$ & 243.2 & 238.1 & 236.9 & 243.5 \\
$\mathrm{Al}-\mathrm{O}-\mathrm{Si}$ & 131.2 & 134.8 & 136.8 & 133.8 \\
\hline \hline
\end{tabular}

terms). When calculating these four deprotonation energies we use four different di-tetrahedra models with $\mathrm{O}(1)-\mathrm{O}(4)$ as bridging atoms, while in reality the anion formed on proton removal from $\mathrm{O}(1)-\mathrm{O}(4)$ sites is always the same. If the QM-Pot scheme worked perfectly, they should attain the same total energy and there should be no differences between the calculated relative stabilities and the relative values of the deprotonation energies. This provides an internal consistency check and indicates that the error we make is of the order of $3 \mathrm{~kJ} / \mathrm{mol}$. Similar calculations on di-tetrahedra clusters embedded differently at symmetry equivalent positions in the faujasite structure showed that the error of the energy is about $\pm 2.5 \mathrm{~kJ} / \mathrm{mol}^{6.8}$

Table II also shows relative stabilities of the proton positions $\mathrm{O} 1-\mathrm{H}$ to $\mathrm{O} 4-\mathrm{H}$ calculated with the periodic HartreeFock method at the structures obtained with the combined QM-Pot method for the embedded di-tetrahedra models. The order of relative stabilities is the same as for the QM-Pot energies which shows the correct performance of the QMPot scheme. The relative stabilities predicted by the periodic HF method deviate by $3-5 \mathrm{~kJ} / \mathrm{mol}$ from respective QM-Pot values.

With respect to the proton siting in chabazite we conclude that without doubt $\mathrm{O} 1-\mathrm{H}$ is the most stable site, while the calculations are not accurate enough to decide whether $\mathrm{O} 3$ or $\mathrm{O} 4$ is second in stability. Experiments on H-SSZ-13 yield $\mathrm{O} 1-\mathrm{H}$ and $\mathrm{O} 3-\mathrm{H}$ as the most stable sites ${ }^{41}$ and a recent density functional theory (DFT) study reports the stability sequence $\mathrm{O} 1-\mathrm{H}>\mathrm{O} 3-\mathrm{H}>\mathrm{O} 2-\mathrm{H}>\mathrm{O} 4-\mathrm{H}^{24}$

\section{B. QM-Pot results for $\mathrm{NH}_{3}$ adsorption}

\section{Initial adsorption positions}

In the neutral adsorption complex, ammonia was attached to the $\mathrm{O} 1-\mathrm{H}$ site and the structure relaxed by use of the shell model potential. For the ion pair structure $\left(\mathrm{NH}_{4}^{+}\right.$-chabazite) different start structures were tried and initial shell model optimizations performed at constant pressure. The $\mathrm{NH}_{4}^{+}$ion is able to form various adsorption complexes which involve either two or three hydrogen bonds to framework oxygen atoms (see Ref. 19 and the references therein) and the small eight-ring pore offers many coordination possibilities. The ammonium ion was placed into the eight-ring twofold coordinated to $\mathrm{O} 1$ and $\mathrm{O} 2$, threefold coordinated to $\mathrm{O} 1, \mathrm{O} 2$, and $\mathrm{O} 3$, or threefold coordinated to $\mathrm{O} 1$, $\mathrm{O} 2$, and $\mathrm{O} 4$. In the most stable conformation the $\mathrm{NH}_{4}^{+}$ion is twofold coordinated to $\mathrm{O} 1$ and $\mathrm{O} 2$ with $\mathrm{O} \cdots \mathrm{H}$ distances of 184 and $193 \mathrm{pm}$, respectively. The lattice constants of this conformation (Table I are close to the lattice constants of $a$ $=9.421 \AA$ and $\alpha=94.2^{\circ}$ from a single crystal x-ray determination of a natural $(\mathrm{Ca}, \mathrm{Sr})$-chabazite. ${ }^{40} \mathrm{~A}$ second conformation involving hydrogen bonds of $182 \mathrm{pm}$ to $\mathrm{O} 1$ and of 204 $\mathrm{pm}$ to $\mathrm{O}^{\prime}$ ' was less stable by $2.7 \mathrm{~kJ} / \mathrm{mol}$. Hence, the $\mathrm{O} 1$ site is the preferred site both for binding protons and coordinating $\mathrm{NH}_{4}^{+}$.

\section{QM-Pot calculations}

These shell model optimized structures were used as initial structures in the following QM-Pot optimizations of the 
TABLE IV. Structures obtained by a complete relaxation of eight-membered ring models embedded in a $2 \times 2 \times 1$ supercell of chabazite and in comparison to structures of eight-membered rings embedded in a cell obtained by averaging the framework coordinates over all four primitive cells of the supercell. Given are selected bond lengths (pm) and angles (deg) of the eight-membered ring models.

\begin{tabular}{|c|c|c|c|c|c|c|c|c|}
\hline \multirow{2}{*}{$\begin{array}{l}\text { Distance or } \\
\text { angle }\end{array}$} & \multicolumn{2}{|c|}{$\mathrm{NH}_{4}-\mathrm{CHA}$} & \multicolumn{2}{|c|}{$\mathrm{NH}_{3}-\mathrm{CHA}$} & \multicolumn{2}{|c|}{$\mathrm{H}-\mathrm{CHA}$} & \multicolumn{2}{|c|}{$\mathrm{CHA}^{-}$} \\
\hline & relaxed & avgd & relaxed & avgd & relaxed & avgd & relaxed & avgd \\
\hline $\mathrm{O} 1-\mathrm{Hb}$ & & & 102.7 & 106.5 & 95.5 & 95.8 & & \\
\hline $\mathrm{Al}-\mathrm{O} 1$ & 178.6 & 180.1 & 186.5 & 188.4 & 191.3 & 191.4 & 173.6 & 174.7 \\
\hline $\mathrm{Si}-\mathrm{O} 1$ & 161.1 & 160.5 & 167.4 & 167.5 & 170.4 & 169.3 & 157.4 & 157.4 \\
\hline $\mathrm{A} 1-\mathrm{O} 2$ & 172.1 & 174.8 & 170.1 & 171.0 & 169.3 & 171.3 & 171.7 & 172.8 \\
\hline $\mathrm{Si}-\mathrm{O} 2$ & 157.4 & 158.4 & 157.7 & 157.7 & 158.4 & 158.6 & 156.5 & 156.9 \\
\hline $\mathrm{Si}-\mathrm{O}^{\prime}$ & 163.5 & 163.2 & 162.4 & 162.7 & 161.9 & 162.1 & 162.0 & 162.0 \\
\hline $\mathrm{Hb}-\mathrm{N}$ & & & 158.6 & 158.9 & & & & \\
\hline $\mathrm{H} 1-\mathrm{O} 1$ & 165.6 & 164.7 & & & & & & \\
\hline $\mathrm{H} 2-\mathrm{O}^{\prime}$ & 219.8 & 222.3 & 255.7 & 255.1 & & & & \\
\hline $\mathrm{N}-\mathrm{O} 1$ & 269.8 & 269.0 & 261.1 & 264.6 & & & & \\
\hline $\mathrm{N}-\mathrm{H} 1$ & \multicolumn{2}{|c|}{104.4} & & & & & & \\
\hline $\mathrm{N}-\mathrm{H} 2$ & \multicolumn{2}{|c|}{101.6} & \multicolumn{2}{|c|}{100.6} & & & & \\
\hline $\mathrm{N}-\mathrm{H} 3$ & \multirow{2}{*}{\multicolumn{2}{|c|}{$\begin{array}{l}101.3 \\
101.5\end{array}$}} & \multicolumn{2}{|c|}{101.1} & & & & \\
\hline $\mathrm{N}-\mathrm{H} 4$ & & & \multicolumn{2}{|c|}{101.0} & & & & \\
\hline $\mathrm{H} 1-\mathrm{N}-\mathrm{H} 2$ & \multicolumn{2}{|c|}{111.5} & & & & & & \\
\hline $\mathrm{Al}-\mathrm{O} 1-\mathrm{Si}$ & 132.0 & 133.5 & 129.9 & 132.7 & 131.5 & 135.8 & 147.7 & 147.7 \\
\hline $\mathrm{O} 1-\mathrm{Hb}-\mathrm{N}$ & & & 176.7 & 173.1 & & & & \\
\hline
\end{tabular}

ion-pair (IP), the neutral complex (NC), the protonated and the deprotonated chabazites. An eight-membered aluminosilicate ring was defined as the QM part which includes all relevant short-range interactions between the adsorbates and the framework. This model is too large to be embedded in a single primitive unit cell, because the link atoms, i.e., the hydrogen atoms of the terminating hydroxyl groups, would extend over the cell border and overlap with the periodic image of the terminal hydroxyl groups at the opposite side of the cluster. Therefore, we constructed a $2 \times 2 \times 1$ supercell which repeats the primitive cells two times in the [100] and [010] directions and which is large enough to host the eightring model in the lower left corner, see Fig. 1.

Table IV summarizes selected bond lengths and angles of the QM-Pot optimized structures. Figure 2 shows the corresponding adsorbate loaded embedded models and compares them with gas-phase clusters. The most stable structure of the $\mathrm{NH}_{4}^{+}$ion is a twofold coordination to $\mathrm{O} 1$ and $\mathrm{O}^{\prime}$ (Figs. 1 and 2), which was only second in stability when the interatomic potential was applied only (vide supra). The three ammonium ions in the other three $1 \times 1 \times 1$ subcells of the $2 \times 2 \times 1$ supercell which are still described by the shell model potential only coordinate to $\mathrm{O} 1$ and $\mathrm{O} 2$. $\mathrm{The}^{+} \mathrm{NH}_{4}^{+}$ion which is described by the QM-Pot method involves hydrogen bond lengths of 166 and $220 \mathrm{pm}$ to $\mathrm{O} 1$ and $\mathrm{O3}^{\prime}$, respectively. The $\mathrm{N}-\mathrm{O}$ distance is $270 \mathrm{pm}$. A much shorter $\mathrm{N}-\mathrm{O}$ distance of 225/pm was found in the CRYSTAL study of Teunissen ${ }^{21}$ and the EMBED study of Pisani,${ }^{27}$ which is probably due to fact that the authors were unable to optimize structure parameters other than the $\mathrm{N}-\mathrm{O}$ distance and applied a minimal basis set. Our results for $\mathrm{NH}_{4}^{+}$-chabazite seem to be more reliable, since a large basis set was applied and the structure was completely relaxed.

Experimental structure data on $\mathrm{NH}_{4}^{+}$-chabazite are not available. A recent single crystal $\mathrm{x}$-ray diffraction study of an anhydrous $\mathrm{NH}_{4}^{+}$-natrolite containing flexible eight-rings reports hydrogen bond lengths between 186 and $208 \mathrm{pm}$, and $\mathrm{N}-\mathrm{O}$ distances between 282 and $287 \mathrm{pm} .{ }^{42}$ A powder $\mathrm{x}$-ray diffraction study of zeolite $\mathrm{NH}_{4}-\mathrm{RHO}$ which also possesses flexible eight-rings yielded $\mathrm{N}-\mathrm{O}$ distances of 289 and 314 $\mathrm{pm}^{43}$

For the $\mathrm{NH}_{3}$ molecule we find a similar adsorption struc-
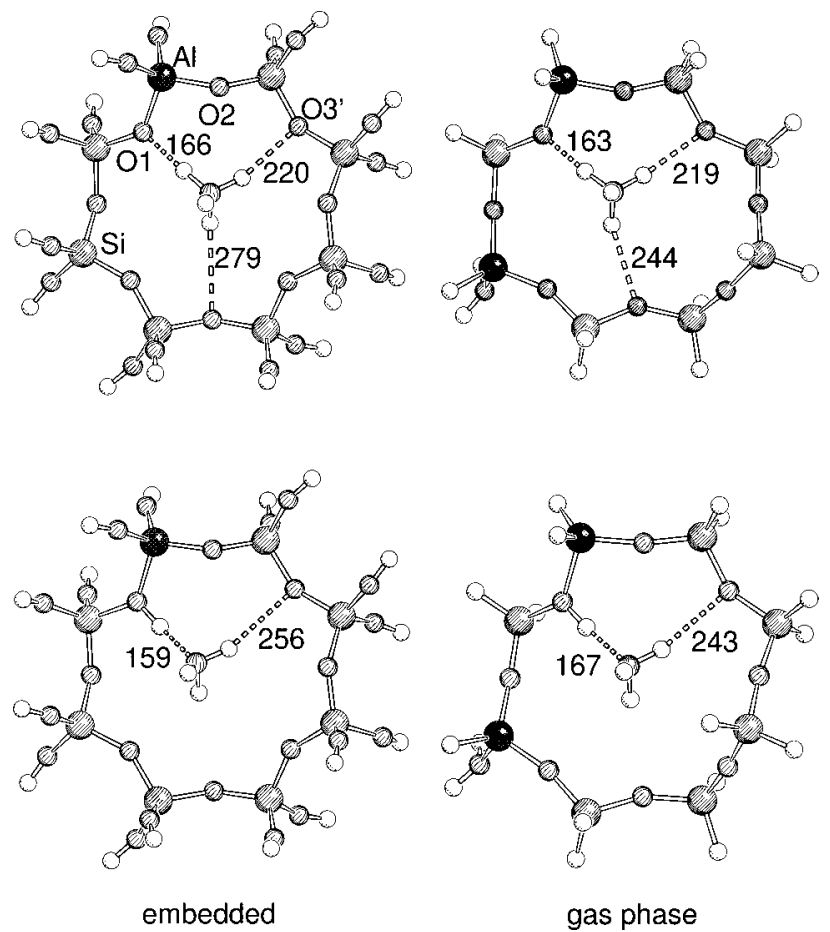

FIG. 2. $\mathrm{NH}_{4}^{+}$and $\mathrm{NH}_{3}$ loaded eight-membered ring models embedded in the chabazite framework and optimized in gas phase. Hydrogen bond lengths in pm. Top row: Ion pair. Bottom row: Neutral complex. 
ture as for $\mathrm{NH}_{4}^{+} . \mathrm{NH}_{3}$ forms a strong H-bond with the $\mathrm{O} 1-\mathrm{H}$ group and points with one of its protons to the $3^{\prime}$ framework site.

\section{Average structures for periodic Hartree-Fock calculations}

Unfortunately, periodic Hartree-Fock calculations are not feasible on the $2 \times 2 \times 1$ unit cell due to their extreme computational cost (144-164 atoms, about 3000 basis functions). Therefore, the following procedure was adopted:

(i) Generation of average primitive unit cells from the coordinates of the QM-Pot optimized $2 \times 2 \times 1$ supercells,

(ii) periodic Hartree-Fock single-point calculations for these average primitive unit cells,

(iii) QM-Pot single-point calculations on eight-ring models embedded in the $2 \times 2 \times 1$ supercells that are obtained from the averaged primitive cells.

This way, structural differences are excluded from the comparison of the periodic HF and the QM-Pot results.

One possibility to construct an average $1 \times 1 \times 1$ cell from the QM-Pot fractional coordinates of the $2 \times 2 \times 1$ supercell is to use the coordinates of the primitive cell in the lower left corner of the $2 \times 2 \times 1$ supercell in which the eight-ring model was embedded. Such an approach is, however, connected with the difficulty of finding coordinates for the atoms lying at the border of the primitive cell, since the oxygen atoms of the terminal hydroxyl groups were allowed to move within the $2 \times 2 \times 1$ unit cell in the QM-Pot optimization and were not subject to translational periodicity of the primitive cell. This poses also difficulties in finding reasonable lattice constants for the primitive cell. It is easier to use the lattice constants of the supercell, to fold back the fractional coordinates of all four primitive cells in the supercell and average them to a single primitive cell. This works well for the framework atoms. Due to the good quality of the $a b$ initio shell model potential for the zeolite only small differences are observed between $\mathrm{T}-\mathrm{O}$ distances obtained by the $a b$ initio shell model and the HF method. ${ }^{6,8,31}$ In contrast, the adsorbates in the embedded eight-rings undergo large motions in the QM-Pot optimization with respect to their position in the Pot part. Hence, averaging over all four adsorbate molecules would lead to large deviations of the averaged structure from the QM-Pot structure. Therefore, only the coordinates of the framework atoms were averaged and the adsorbate coordinates of the QM part were used. Table IV compares bond lengths and angles for the averaged and the original structures. The averaging introduces maximum changes in the $\mathrm{Si}-\mathrm{O}$ and $\mathrm{Al}-\mathrm{O}$ bond lengths of 1.6 and 2.7 $\mathrm{pm}$, respectively. The $\mathrm{O}-\mathrm{H} b$ distance in the ammonia structure changes by $3.8 \mathrm{pm}$, since the position of the acidic proton was not averaged due to big differences of the proton positions in the Pot and QM part. ${ }^{44}$ The lengths of the hydrogen bonds of the adsorbates with the framework oxygens given in Table IV changed by no more than $2.5 \mathrm{pm}$. Larger changes of 4-10 pm were observed for the hydrogen bonds longer than $260 \mathrm{pm}$. For the reaction energies we will expect, therefore, the biggest changes introduced by the averaging
TABLE V. Reaction energies $(\mathrm{kJ} / \mathrm{mol})$ obtained from ab initio calculations on gas-phase eight-ring models containing two $\mathrm{Al}$ atoms (see Fig. 2), on eight-ring models embedded in $2 \times 2 \times 1$ supercells of chabazite (relaxed and averaged frameworks), and on the periodic chabazites (averaged frameworks). The energies for the embedded and periodic structures are given per primitive unit cell.

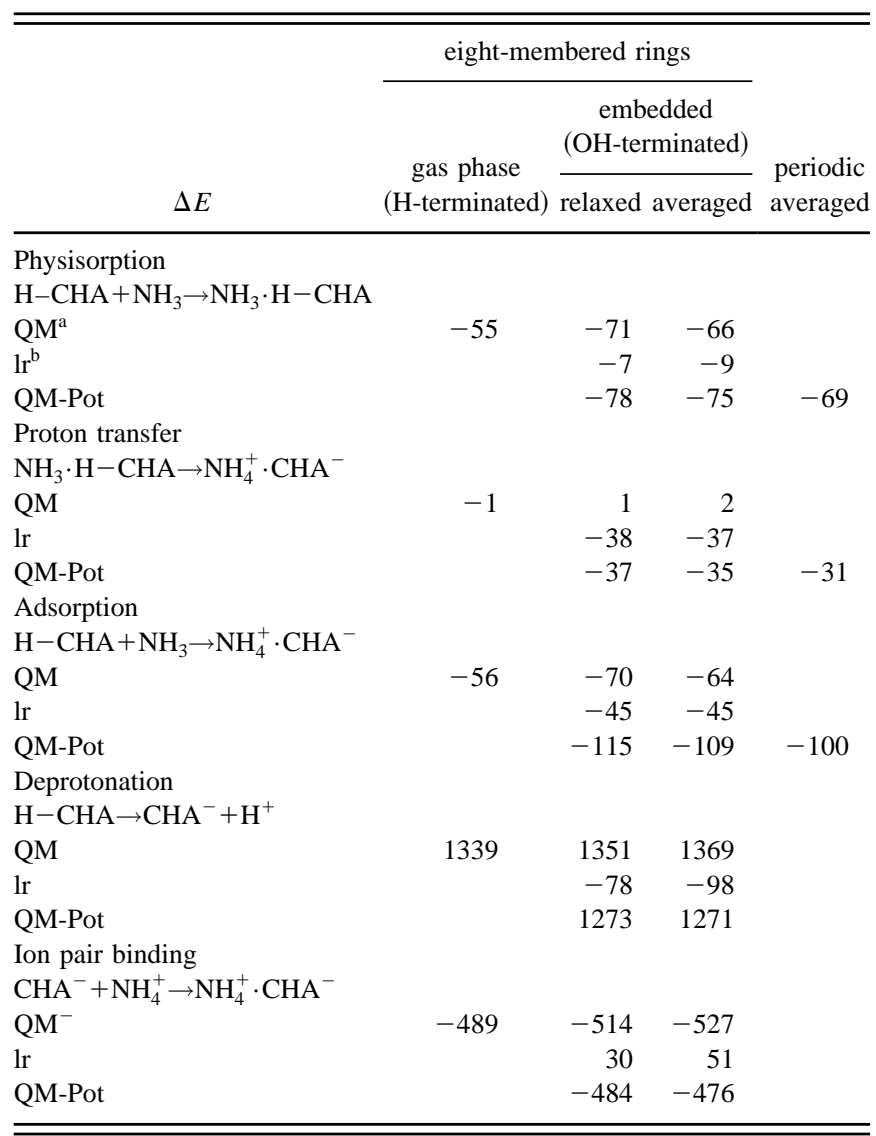

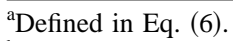

${ }^{\mathrm{b}}$ Defined in Eqs. (7)-(9).

where the adsorbate loaded structures are involved in the reaction.

\section{Reaction energies and effect of averaging}

Table V presents energies of the reactions of Scheme 1 obtained by periodic Hartree-Fock calculations for the averaged primitive cells [step (ii)], and by QM-Pot calculations for eight-ring models embedded in $2 \times 2 \times 1$ supercells [step (iii)]. All energies are defined per single primitive unit cell. The long-range contribution for the averaged cell structure can be calculated exactly as

$$
\begin{gathered}
\Delta E_{\mathrm{lr} / / \mathrm{QM}-\mathrm{Pot}}(1 \times 1 \times 1)=\Delta E_{\mathrm{lr} / / \mathrm{QM}-\mathrm{Pot}}(2 \times 2 \times 1) \\
-3 * \Delta E_{\mathrm{Pot}}(1 \times 1 \times 1), \\
\Delta E_{\mathrm{Pot}}(1 \times 1 \times 1)=E_{\mathrm{Pot}}\left(S_{P}, 1 \times 1 \times 1\right) \\
-E_{\mathrm{Pot}}\left(S_{R}, 1 \times 1 \times 1\right) .
\end{gathered}
$$

This is equivalent to dividing the contribution of the periodic systems in Eq. (7) by four. For the relaxed $2 \times 2 \times 1$ supercells, we approximate the $1 r$ contribution per $1 \times 1 \times 1$ cell by using the shell model start structures of the $1 \times 1 \times 1$ cells. I.e., we assume that the structures of the three averaged 
primitive cells described by the shell model only did not change considerably with respect to the start structures.

The energetic effects of averaging the $2 \times 2 \times 1$ supercell structure can be judged on comparison of the QM-Pot results for the eight-ring models embedded in the relaxed and averaged cells (Table V). In the case of deprotonation we find excellent agreement between the combined QM-Pot results, while the QM and the long-range contributions alone are more sensitive to averaging the structure. The largest absolute deviation in the QM-Pot energies, $8 \mathrm{~kJ} / \mathrm{mol}$, is observed for the ion pair binding energy. For the key reactions, adsorption, and proton transfer, the differences are 6 and 2 $\mathrm{kJ} / \mathrm{mol}$, respectively, only about $5 \%$.

\section{E. Electrostatic potential maps}

For the success of the combined QM-Pot approach it is vital that the subtraction scheme, Eq. (1), approximates closely the correct electrostatic potential (ESP) in the reaction area, i.e., inside the zeolite cavity. In this section, we compare the ESP calculated within the QM-Pot scheme with its periodic HF limit. According to Eq. (1), the QM-Pot scheme adds a correction to the ESP calculated from the wave function of the embedded cluster, which is defined as the difference between the ESP of the periodic host and the embedded cluster both calculated with the $a b$ initioparametrized shell model potential.

$$
\mathrm{ESP}_{\mathrm{QM}-\mathrm{Pot}}(S)=\mathrm{ESP}_{\mathrm{QM}}(C)+\mathrm{ESP}_{\mathrm{Pot}}(S)-\mathrm{ESP}_{\mathrm{Pot}}(C) .
$$

The electrostatic potential of the protonated chabazite was calculated at a grid of 2601 points in a plane passing through the eight-ring in the manner that it contained the bridging hydroxyl group. No further approximations such as use of multipolar expansions ${ }^{37}$ were made. Figure 3 shows the ESP maps of the periodic system $(S)$ and the embedded cluster $(C)$ as well as the respective difference maps $\operatorname{ESP}(S)$ $-\operatorname{ESP}(C)$ calculated with both QM and shell model potential methods. The map at the bottom shows the combined QM-Pot result obtained according to Eq. (10). Since surface terms due to lattice sum truncation in the periodic calculations introduce a constant potential shift, the ESP from the periodic HF calculation was shifted by an offset for the purpose of comparison. This shift was set so that the differences $\operatorname{ESP}_{\mathrm{QM}}(S)-\operatorname{ESP}_{\mathrm{Pot}}(S)$ and $\mathrm{ESP}_{\mathrm{QM}}(C)-\operatorname{ESP}_{\mathrm{Pot}}(C)$ were the same at the center point.

Whereas the ESP calculated from the wave functions is always positive in the core region, the shell model ESP for the periodic zeolite and the cluster model exhibit positive and negative maxima due to the use of formal charges. Within the eight-ring pore the curvature of the contour lines is very similar for both the HF and shell model descriptions. The regions of negative potential are close to the oxygen atoms. These are the energetically most favorable regions for interactions with positively charged species. The attraction is larger in the case of the shell model. Figures 4(a) and 4(b) show cuts of $\operatorname{ESP}(S)$ and the difference $\operatorname{ESP}(S)-\operatorname{ESP}(C)$ in the plane along a diagonal between the acidic proton and the opposite oxygen of the eight-ring pore. In Fig. 4(a), the gradient of the shell model ESP around the acidic proton is

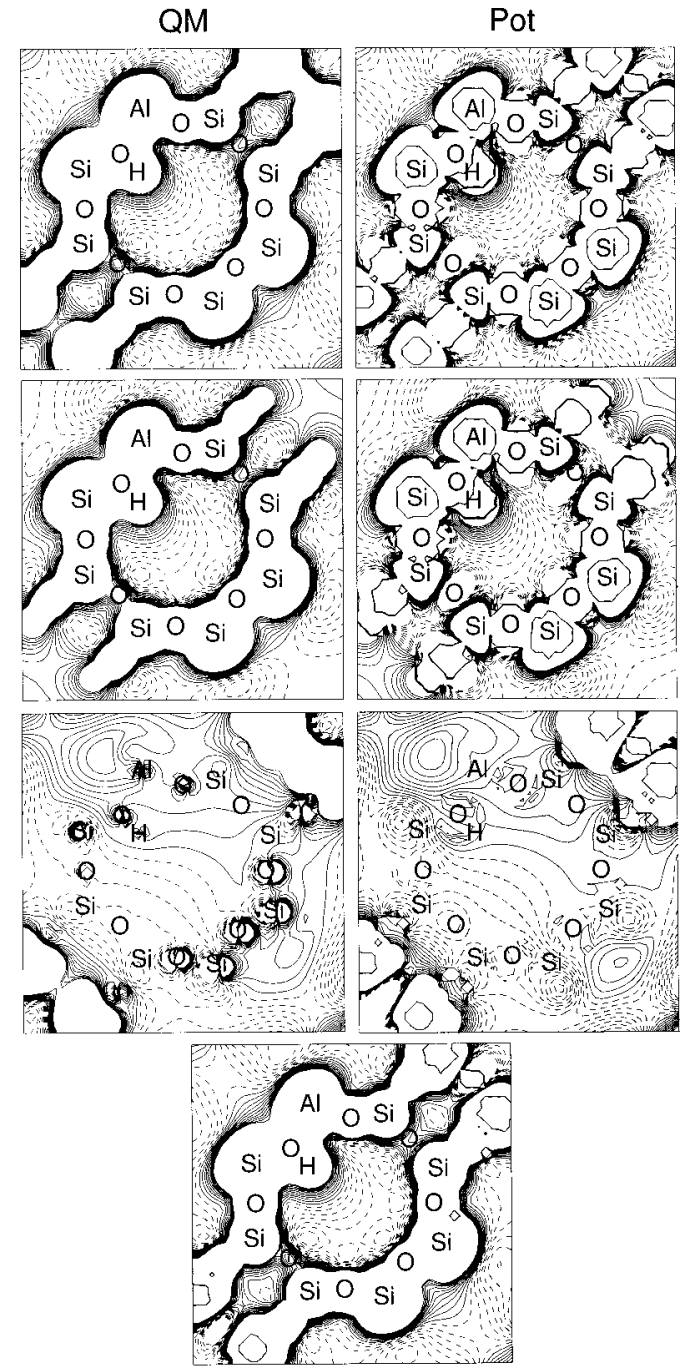

FIG. 3. Electrostatic potential (ESP) in a plane passing through the eightring pore of acidic chabazite and containing the bridging hydroxyl group. Left column: Hartree-Fock (QM) calculations. Right column: Shell model potential (Pot) calculations. First row: Periodic framework. (The QM ESP is shifted by 0.1784 a.u.). Second row: Eight-ring model. Third row: Periodic framework minus eight-ring model. Fourth row: QM-Pot scheme, $\mathrm{QM}-\operatorname{Pot}(S)=\mathrm{QM}(C)+\operatorname{Pot}(S)-\operatorname{Pot}(C)$. Solid lines indicate positive values, dashed lines negative values of the ESP. Equidistance of lines 0.01 a.u. $(0.272 \mathrm{~V})$ in rows $1,2,4$. Lines corresponding to absolute values higher than 0.1 a.u. are not drawn. Consecutive lines in difference maps in row three differ by 0.005 a.u. $(0.136 \mathrm{~V})$. There, lines corresponding to absolute values higher than 0.05 a.u. are not drawn.

steeper than that from the ab initio model. Obviously the electric field in the pore is overestimated by formal charge models, which was also observed in recent studies of siliceous zeolites. ${ }^{37,45}$ The differences $(S)-(C)$ in Figs. 3 and 4(b) show a shallow sweep in the pore region, which demonstrates that a big enough cluster model is able to account already for a large part of the electrostatic potential at the active site. Both QM and Pot maps are very similar within the pore region. This justifies the use of the ESP difference periodic system-embedded cluster from the shell model as perturbative long-range correction. Addition of this correction to the SCF ESP of the embedded cluster yields the QMPot ESP shown at the bottom of Fig. 3 which is close to the ESP of the periodic QM calculation. We conclude that the $a b$ 
a)

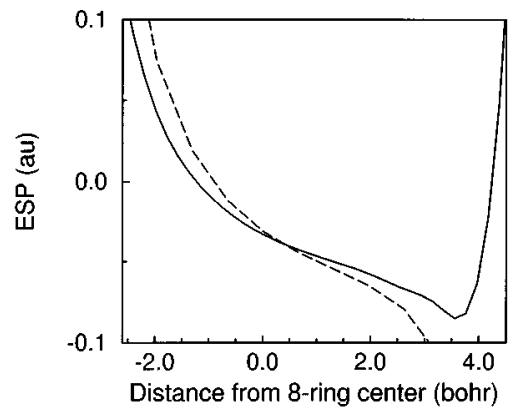

b)

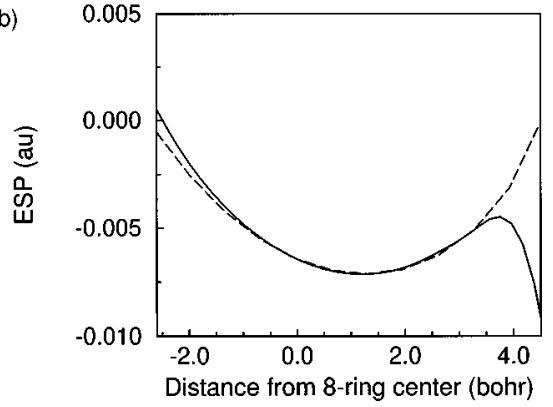

FIG. 4. Electrostatic potential (ESP) within the eight-ring pore along a diagonal passing the plane in Fig. 3 from the top left to the bottom right. (a) $\operatorname{ESP}(S)$. (b) Difference $\operatorname{ESP}(S)-\operatorname{ESP}(C)$. Solid lines: ESP from HartreeFock (QM) calculations. Dashed lines: ESP from ab initio shell model potential (Pot) calculations.

initio-parametrized shell model potential provides a good approximation to the long-range corrections with the tendency to overestimate these contributions to the interaction energy.

\section{F. Comparison of QM-Pot energies with periodic Hartree-Fock results}

For the three reactions that do not involve charged unit cells, physisorption of ammonia, adsorption of ammonia yielding ammonium ions, and proton transfer from the neutral physisorption structure to the ion pair adsorption structure, Table V shows the periodic Hartree-Fock energies obtained for the averaged structures. The combined QM-Pot energies for the same structures are also given. They are all about $10 \%$ larger in absolute terms. This fits to the results for the electrostatic potentials presented in the previous section. The largest difference, $9 \mathrm{~kJ} / \mathrm{mol}$, is found for the chemisorption energy and the smallest difference, $4 \mathrm{~kJ} / \mathrm{mol}$, is observed for the proton transfer energy. Given the accuracy of the periodic Hartree-Fock calculations of $\pm 1 \mathrm{~kJ} / \mathrm{mol}$, this is an excellent confirmation of the soundness of the QM-Pot scheme. The deviation is expected to become even smaller when larger clusters are used for the quantum part since an increasing share of the long-range contribution is evaluated quantum mechanically. In previous applications of the QMPot scheme the QM contribution was indeed found to increase and the long-range contribution to decrease with increasing size of the embedded cluster. ${ }^{7-9}$ For the zeolites with large unit cells faujasite, ZSM-5, and mordenite the total QM-Pot reaction energies of $\mathrm{NH}_{3}$ adsorption were stable within $\pm 3 \mathrm{~kJ} / \mathrm{mol}$ with respect to changes of cluster size and shape. ${ }^{7-9}$ Because of the composition and the small
TABLE VI. Ellipticities (pm) of eight-membered rings embedded in the $2 \times 2 \times 1$ supercell of chabazite and optimized in gas phase. The ellipticities are defined as difference of each two perpendicular diagonals connecting two opposite oxygen atoms. Set 1: O2 sites, set 2: O1 and O3 sites.

\begin{tabular}{|c|c|c|}
\hline & Embedded & Free space model \\
\hline Structure & diagonal set $1 /$ set 2 & diagonal set $1 /$ set 2 \\
\hline $\mathrm{NH}_{4}-\mathrm{CHA}$ & $31 / 52$ & $46 / 156$ \\
\hline $\mathrm{NH}_{3}-\mathrm{CHA}$ & $25 / 47$ & $35 / 48$ \\
\hline $\mathrm{H}-\mathrm{CHA}$ & $30 / 14$ & $53 / 122$ \\
\hline $\mathrm{CHA}^{-}$ & $24 / 4$ & $33 / 10$ \\
\hline
\end{tabular}

size of the unit cell of chabazite (necessary to be able to complete the periodic HF calculations) the embedded cluster cannot be made larger if comparison with the fully periodic quantum calculation is the aim. The details are outlined in Ref. 9. Moreover, for small unit cells the interactions between the adsorbates or active sites in neighboring unit cells are not negligible, as discussed in Sec. III. Hence, we expect that for zeolites with larger unit cells the comparison between QM-Pot and fully periodic QM energies would be even more favorable if the latter could be completed.

\section{G. Comparison with previous work}

Experimentally there is not doubt that in agreement with the present predictions on adsorption of ammonia in acidic zeolites the acidic proton is transferred onto ammonia and ammonium ions are formed. ${ }^{15-18}$ Detailed comparison of the QM-Pot chemisorption energy with microcalorimetric data was already made in Ref. 9. Here we focus on comparison with previous calculations.

First, comparison will be made with results obtained for eight-ring models optimized in the gas phase without any constraints. ${ }^{19}$ They differ from the embedded clusters of the present study in two respects. They are terminated by $\mathrm{H}$ atoms instead of $\mathrm{OH}$ groups and contain two $\mathrm{Al}$ atoms instead of one. This low Si/Al ratio is the same as chosen by Teunissen et al. in their periodic HF study. ${ }^{21}$ Figure 2 shows the structures of the neutral $\left(\mathrm{NH}_{3}\right)$ and ion pair $\left(\mathrm{NH}_{4}^{+}\right)$adsorption complexes in comparison with the combined QM-Pot results for the embedded clusters. Even if the $\mathrm{Si} / \mathrm{Al}$ ratios are different, comparison of the embedded and the free space models provides an idea how the constraints imposed by the zeolite framework affect the structure relaxation at the reaction site. The embedded eight-ring models remain nearly circular, whereas the gas-phase models wrap around the adsorbates due their high flexibility and the lack of constraints. A measure for the distortion are the approximate eight-ring ellipticities calculated from the difference of each two perpendicular diagonals connecting opposite oxygen atoms in the eight-ring (Table VI). They are always larger for the free than for the embedded eight-rings. Especially large values are observed for the ion-pair and the protonated structures. The distortions in these models are caused by the tendency of the framework oxygen atoms to form hydrogen bonds with the protons of the $\mathrm{NH}_{4}^{+}$ion or with the acidic proton, respectively. 
Table $\mathrm{V}$ includes the reaction energies calculated for the free space eight-ring models. In spite of the differences between the free space and the embedded models, the results are qualitatively the same. The IP structure $\left(\mathrm{NH}_{4}^{+}\right)$is slightly more stable than the $\mathrm{NC}$ structure $\left(\mathrm{NH}_{3}\right)$ but the difference is only $1 \mathrm{~kJ} / \mathrm{mol}$. The adsorption energy of the free cluster, $-56 \mathrm{~kJ} / \mathrm{mol}$, is smaller than that of the embedded cluster, $-109 \mathrm{~kJ} / \mathrm{mol}$, and even slightly smaller than the QM contribution of the embedded cluster.

Second, we comment on previous periodic HartreeFock calculations ${ }^{21}$ and electronic embedding calculations based on Green's function techniques. ${ }^{27}$ In these calculations the neutral adsorption complex was found to be more stable than the ion pair complex by as much as $100-200 \mathrm{~kJ} / \mathrm{mol}$. In contrast, both our periodic HF and our combined QM-Pot calculations agree on the prediction that the ion pair complex with the $\mathrm{NH}_{4}^{+}$ion is the stable product when $\mathrm{NH}_{3}$ is adsorbed in H-chabazite. It is by $30-35 \mathrm{~kJ} / \mathrm{mol}$ more stable than the neutral physisorption structure. Both of the previous studies employed a minimal basis set (STO-3G) and assumed rigid zeolite frameworks and adsorbates. The $\mathrm{N}-\mathrm{O}$ distance was the only degree of freedom optimized. One of us has outlined before ${ }^{19}$ that the reason for the unrealistic results is the use of the STO-3G basis set which is not adequate for anions and yields deprotonation energies that are by far too large.

To check the influence of neglected structure relaxation onto the stability of the IP structure we adopted a similar procedure as Teunissen et al. ${ }^{21}$ We took our optimized $\mathrm{NH}_{3}-\mathrm{CHA}$ structure and transferred the acidic proton along the $\mathrm{Ob}-\mathrm{N}$ axis to the ammonia molecule. The $\mathrm{N}-\mathrm{H}$ bond length of the $\mathrm{NH}_{4}^{+}$ion formed this way was set to $101 \mathrm{pm}$. A single point QM-Pot calculation was then carried out for this distorted ion pair using the same $\mathrm{T}(\mathrm{O}, \mathrm{N}) \mathrm{DZP}$ basis set as in all other calculations of this study. The QM proton transfer energy calculated from this structure is $+34 \mathrm{~kJ} / \mathrm{mol}$, which is way below Teunissen's periodic STO-3G result of +194 $\mathrm{kJ} / \mathrm{mol}^{21}$ The long-range contribution is $-39 \mathrm{~kJ} / \mathrm{mol}$, virtually identical with the $-38 \mathrm{~kJ} / \mathrm{mol}$ we obtained for the fully relaxed structure. For the proton transfer reaction we obtain a combined QM-Pot energy of $-5 \mathrm{~kJ} / \mathrm{mol}$, which shows preferred stability of the IP over the NC even for a heavily constraint structure. We conclude that use of flexible basis sets which describe anions properly is mandatory. Errors connected with rigid zeolite structures are significantly smaller. We also note that in the constraint structure the $\mathrm{NH}_{4}^{+}$ion is coordinated to one framework oxygen only, and still we get the lowest energy for the ion-pair structure. This is contrary to previous ideas that two or threefold coordination may be key for the proton transfer to happen. We have shown that as long as a proper basis set is used and longrange effects are taken into account the ion pair complex is the most stable structure even if it is heavily constraint.

A final comment concerns other embedding schemes which embed the cluster into an array of charge or multipoles. They permit structure optimization if at all only for an inner region of the quantum part while the positions of the sites defining the external electrostatic potential are fix. ${ }^{25,26,46,47}$ In contrast, the present embedding scheme is the only one allowing for a complete relaxation of the periodic zeolite structure. The accuracy of the structure predictions makes it a useful tool if fully periodic ab initio calculations are feasible just for single structures. Even if fully periodic $a b$ initio structure optimizations are feasible, the present scheme can provide excellent start structures which speed up such calculations significantly.

\section{CONCLUSIONS}

In agreement with experiments, in H-chabazite the preferred proton site is framework oxygen $\mathrm{O} 1$. On adsorption of $\mathrm{NH}_{3}$ the acid site proton is transferred onto $\mathrm{NH}_{3}$ and a $\mathrm{NH}_{4}^{+}$ ion is formed which is coordinated to two framework oxygen atoms of the eight-membered alumosilicate ring. The energy of this ion-pair structure is lower than that of the neutral adsorption complex by about $35 \mathrm{~kJ} / \mathrm{mol}$. To correctly predict the relative energies of the two structures it is more important to use an adequate basis set than to relax the structure of the zeolite completely.

Comparison is made between a combined QMinteratomic potential function approach and a fully periodic quantum-mechanical treatment, both at the Hartree-Fock level. While the former is also suitable for optimizing structures, the latter is practical for single point energy calculations only. The small differences between energies calculated with the two methods of the order of 4-9 $\mathrm{kJ} / \mathrm{mol}$ strongly supports the combined QM-Pot approach. Comparison of the electrostatic potentials calculated with the two methods shows that the use of an $a b$ initio-parametrized interatomic potential function that takes electronic polarization into account (shell model) is crucial for the success of the approach. The possibility to fully relax periodic structures of solids with large unit cells is the advantage of the present embedded cluster approach compared to other schemes.

Note added in proof. A paper has been overlooked ${ }^{48}$ which compares the periodic STO-3G calculations ${ }^{21}$ with a cluster model embedded in an array of potential derived point charges. ${ }^{46}$ The conclusion is reached that this simple scheme shows better agreement with the periodic calculation than the more sophisticated embedding scheme used in Ref. 27 , at least for the STO-3G basis set.

\section{ACKNOWLEDGMENTS}

We thank J. D. Gale (London) and R. Ahlrichs (Karlsruhe) for providing recent versions of the GULP and TURBOMOLE codes, respectively. M.B. acknowledges a research fellowship of the Alexander-von-Humboldt foundation and thanks René Windiks for technical assistance in the $\mathrm{H}-\mathrm{CHA}$ calculations. This work has been supported by the "Fonds der Chemischen Industrie" and by the "MaxPlanck-Gesellschaft."

${ }^{1} \mathrm{~J}$. Gao, in Reviews in Computational Chemistry, edited by K. B. Lipkowitz and D. B. Boyd (VCH, New York, 1996), Vol. 7, p. 119.

${ }^{2}$ R. V. Stanton, D. S. Hartsough, and K. M. Merz, Jr., J. Comput. Chem. 16, 113 (1995).

${ }^{3}$ R. V. Stanton, D. S. Hartsough, and K. M. Merz, Jr., J. Phys. Chem. 97, 11868 (1993).

${ }^{4}$ A. Warshel, Computer Modelling of Chemical Reactions in Enzymes and Solutions (Wiley, New York, 1991). 
${ }^{5}$ T. Matsubara, F. Maseras, N. Koga, and K. Morokuma, J. Phys. Chem. 100, 2573 (1996).

${ }^{6}$ U. Eichler, C. M. Kölmel, and J. Sauer, J. Comput. Chem. 18, 463 (1996).

${ }^{7}$ M. Brändle and J. Sauer, J. Mol. Catal. A: Chemical 119, 19 (1997).

${ }^{8}$ U. Eichler, M. Brändle, and J. Sauer, J. Phys. Chem. B 101, 10035 (1997).

${ }^{9}$ M. Brändle and J. Sauer, J. Am. Chem. Soc. 120, 1556 (1998).

${ }^{10}$ D. Bakowies and W. Thiel, J. Phys. Chem. 100, 10580 (1996).

${ }^{11}$ C. Pisani, R. Dovesi, and C. Roetti, Hartree-Fock Ab-initio Treatment of Crystalline Systems, Lecture Notes in Chemistry Vol 48 (Springer, Berlin, 1988).

${ }^{12}$ R. Dovesi, V. R. Saunders, C. Roetti, M. Causà, N. M. Harrison, R. Orlando, and E. Aprà, CRYSTAL95 User's Manual (University of Torino, Torino, 1996).

${ }^{13}$ L.-T. Yuen, S. I. Zones, T. V. Harris, E. J. Gallegos, and A. Auroux, Microporous Mater. 2, 105 (1994).

${ }^{14}$ S. I. Zones, T. V. Harris, and A. Auroux (in preparation).

${ }^{15}$ A. L. Blumenfeld, D. Coster, and J. J. Fripiat, J. Phys. Chem. 99, 15181 (1995).

${ }^{16}$ W. P. J. H. Jacobs, J. L. de Haan, L. J. M. van den Ven, and R. A. van Santen, J. Phys. Chem. 97, 10394 (1993).

${ }^{17}$ W. P. J. H. Jacobs, J. H. M. C. van Wolput, and R. A. van Santen, Zeolites 13, 170 (1993).

${ }^{18}$ J. B. Uytterhoeven, L. G. Christner, and W. K. Hall, J. Phys. Chem. 69, 2117 (1965).

${ }^{19}$ J. Sauer, P. Ugliengo, E. Garrone, and V. R. Saunders, Chem. Rev. 94, 2095 (1994).

${ }^{20}$ J. Sauer, Chem. Rev. 89, 199 (1989).

${ }^{21}$ E. H. Teunissen, C. Roetti, C. Pisani, A. J. M. de Man, A. P. J. Jansen, R. Orlando, R. A. van Santen, and R. Dovesi, Modell. Simul. Mater. Sci. Eng. 2, 921 (1994).

${ }^{22}$ R. Shah, J. D. Gale, and M. C. Payne, J. Phys. Chem. 100, 11688 (1996).

${ }^{23}$ F. Haase, J. Sauer, and J. Hutter, Chem. Phys. Lett. 266, 397 (1997).

${ }^{24}$ R. Shah, J. D. Gale, and M. C. Payne, Phase Transit. 61, 67 (1997).

${ }^{25}$ E. H. Teunissen, A. P. J. Jansen, R. A. van Santen, R. Orlando, and R. Dovesi, J. Chem. Phys. 101, 5865 (1994).

${ }^{26}$ E. H. Teunissen, A. P. J. Jansen, and R. A. van Santen, J. Phys. Chem. 99, 1873 (1995).

${ }^{27}$ C. Pisani and U. Birkenheuer, Int. J. Quantum Chem., Quantum Chem. Symp. 29, 221 (1995).

${ }^{28}$ U. Eichler, PhD thesis (Humboldt University, Berlin, 1997).

${ }^{29}$ R. Ahlrichs, M. Bär, M. Häser, H. Horn, and C. Kölmel, Chem. Phys. Lett. 162, 165 (1989); TURBOMOLE is commercially available from Molecular Simulations Inc., San Diego, California.
${ }^{30}$ J. D. Gale, J. Chem. Soc., Faraday Trans. 93, 629 (1997); J. D. Gale, Program GULP (General Utility Lattice Program) (Royal Institution/ Imperial College, London, 1992).

${ }^{31}$ K.-P. Schröder and J. Sauer, J. Phys. Chem. 100, 11043 (1996).

${ }^{32} \mathrm{~S}$. Huzinaga, Approximate atomic wave functions I, II (University of Alberta, Edmonton, 1971); S. Huzinaga, J. Chem. Phys. 42, 1293 (1965).

${ }^{33}$ J. R. Hill and J. Sauer, J. Phys. Chem. 98, 1238 (1994).

${ }^{34}$ B. G. Dick and A. W. Overhauser, Chem. Rev. 112, 90 (1958).

${ }^{35}$ M. Leslie and M. J. Gillan, J. Phys. C 18, 973 (1985).

${ }^{36}$ S. Suhai, P. S. Bagus, and J. Ladik, Chem. Phys. 68, 467 (1982).

${ }^{37}$ V. R. Saunders, C. Freyria-Fava, R. Dovesi, L. Salasco, and C. Roetti, Mol. Phys. 77, 629 (1992).

${ }^{38}$ V. R. Saunders and I. H. Hillier, Int. J. Quantum Chem. 7, 699 (1973).

${ }^{39}$ R. J. Harrison and J. Nieplocha, The TCGMSG Message-Passing Toolkit, Pacific Northwest National Laboratory, Richland WA 99352, http:// www.emsl.pnl.gov:2080/docs/nwchem/tegmsg.html.

${ }^{40}$ M. Calligaris, G. Nardin, L. Randaccio, and P. Comin Chiaramonti, Acta Crystallogr., Sect. B: Struct. Crystallogr. Cryst. Chem. 38, 602 (1982).

${ }^{41}$ L. J. Smith, A. Davidson, and A. K. Cheetham, Catal. Lett. 49, 143 (1997). In this work, the lattice constants are given in the hexagonal setting of the space group R-3m. For comparison with our structures, we give here the corresponding lattice constants transformed to the rhombohedral setting. The authors employ a different oxygen numbering than in earlier work (Ref. 40). We have also transformed this numbering to the convention used in Ref. 40.

${ }^{42}$ E. Stuckenschmidt, D. Kassner, W. Joswig, and W. H. Baur, Eur. J. Mineral. 4, 1229 (1992).

${ }^{43}$ L. B. McCusker, Zeolites 4, 51 (1984).

${ }^{44} \mathrm{As}$ check we have also excluded the $\mathrm{Ob}$ atom from the averaging of the $\mathrm{NH}_{3}$ - CHA structure. For this structure, the QM-Pot and periodic Hartree-Fock energies of physisorption and proton transfer deviate by less than $1 \mathrm{~kJ} / \mathrm{mol}$ from the values given in Table VI.

${ }^{45}$ J. C. White and A. C. Hess, J. Phys. Chem. 97, 6398 (1993).

${ }^{46}$ S. P. Greatbanks, P. Sherwood, and I. H. Hillier, J. Phys. Chem. 98, 8134 (1994).

${ }^{47}$ A. Kyrlidis, S. J. Cook, A. K. Chakraborty, A. T. Bell, and D. N. Theodorou, J. Phys. Chem. 99, 1505 (1995).

${ }^{48}$ S. P. Greatbanks, I. H. Hillier, and P. Sherwood, J. Comput. Chem. 18, 562 (1997). 